\title{
RAS Protein Family Activation Assay
}

National Cancer Institute

\section{Source}

National Cancer Institute. RAS Protein Family Activation Assay. NCI Thesaurus. Code C139305.

An in vitro assay that can indirectly detect the activation of small GT Pases in the RAS family through the staining of phosphorylated forms of MEK (MAP2K) family proteins (pMEK). A farnesylated RAS family protein is anchored to an in vitro model of the plasma membrane and, under different conditions, is co-incubated with both a RAF family protein, which binds to and is activated by active RAS proteins, and a MEK family protein, which is phosphorylated by active RAF proteins. PMEK is detected using a donor bead coated with anti-MEK antibodies that is capable of oxidizing an acceptor molecule attached to an anti-pMEK antibody. When the donor and acceptor are in close proximity, the acceptor molecule is oxidized and can be visualized using electron microscopy. 